\title{
Rational diagnostic strategy for Zellweger syndrome spectrum patients
}

\author{
Cindy Krause ${ }^{1,2}$, Hendrik Rosewich ${ }^{1,2}$ and Jutta Gärtner*,1 \\ ${ }^{1}$ Department of Pediatrics and Pediatric Neurology, Faculty of Medicine, Georg August University, Göttingen, Germany
}

Zellweger syndrome spectrum (ZSS) comprises a clinically and genetically heterogeneous disease entity, which is caused by mutations in any of the 12 different human PEX genes leading to impaired biogenesis of the peroxisome. Patients potentially suffering from ZSS are diagnosed biochemically by measuring elevated levels of very long chain fatty acids, pristanic acid and phytanic acid in plasma and serum and reduced levels of ether phospholipids in erythrocytes. Published reports on diagnostic procedures for ZSS patients are restricted either to biochemical markers or to defined mutations in a subset of PEX genes. Clarification of the primary genetic defect in an affected patient is crucial for genetic counselling, carrier testing or prenatal diagnosis. In this study, we present a rational diagnostic strategy for patients suspected of ZSS. By combining cell biology and molecular genetic methods in an appropriate sequence, we were able to detect the underlying mutation in various PEX genes within adequate time and cost. We applied this method on 90 patients who presented at our institute, Department of Pediatrics and Pediatric Neurology at Georg August University, and detected 174 mutant alleles within six different PEX genes, including two novel deletions and three new missense mutations in PEX6. Furthermore, this strategy will extend our knowledge on genotype-phenotype correlation in various PEX genes. It will contribute to a better understanding of ZSS pathogenesis, allowing the investigation of the effects of diverse mutations on the interaction between PEX proteins and peroxisomal function in vivo.

European Journal of Human Genetics (2009) 17, 741-748; doi:10.1038/ejhg.2008.252; published online 14 January 2009

Keywords: Zellweger syndrome spectrum; peroxisome; diagnosis; PEX mutation

\section{Introduction}

Peroxisomal disorders represent a group of inborn errors of metabolism that are clinically and genetically heterogeneous. ${ }^{1,2}$ Depending on the nature of peroxisomal impairment, these disorders are usually divided into two major groups (I and II). Disturbance of peroxisome biogenesis is the hallmark of the first group (group I), known as the peroxisome biogenesis disorders (PBDs). ${ }^{3}$ It comprises four different diseases, including Zellweger syndrome (ZS),

*Correspondence: Professor J Gärtner, Department of Pediatrics and Pediatric Neurology, Faculty of Medicine, Georg August University, Robert-Koch-Strasse 40, Göttingen 37075, Germany.

Tel: + 49551 398035; Fax: + 49551 396252;

E-mail: gaertnj@med.uni-goettingen.de

${ }^{2}$ These authors contributed equally to this work.

Received 22 May 2008; revised 23 October 2008; accepted 26 November 2008; published online 14 January 2009 neonatal adrenoleukodystrophy (NALD), infantile Refsum disease (IRD), as well as rhizomelic chondrodysplasia punctata (RCDP). ${ }^{4}$ Clinical symptoms of patients with ZS, NALD and IRD (referred together as Zellweger syndrome spectrum (ZSS)) range from severe neurologic dysfunction (muscular hypotonia, failure to thrive, seizures) and typical dysmorphic features (large fontanelle, wide sutures, high forehead, hypertelorism, broad nasal bridge, external ear deformity) to hepatomegaly, as well as renal cysts. Distinct from ZSS, RCDP describes a clinical phenotype with shortening of the proximal limbs, cataracts and profound psychomotor retardation. The second group (group II) is characterized by deficiency of single peroxisomal enzymes/proteins but efficient peroxisome biogenesis. This group includes at least 12 different diseases, with X-linked adrenoleukodystrophy being the most common $^{1}$. 
Diagnosis of both groups has been traditionally based on clinical and biochemical criteria as the first step. However, due to the increasing knowledge on enormous genetic heterogeneity and the broad clinical spectrum, diagnostic work-up is becoming more complicated. As peroxisomes are the site of $\beta$-oxidation of very long chain fatty acids (VLCFAs), ${ }^{4}$ these fatty acids are elevated in body fluids and tissues in the vast majority of patients with peroxisomal disorder. Thus, they are a convenient screening marker for PBD or a single peroxisomal enzyme/protein deficiency ${ }^{1}$ in many patients. However, an unambiguous diagnosis cannot be relied solely on the measurement of VLCFA concentrations in plasma, as patients clearly suffering from a peroxisomal disorder but containing normal concentrations of VLCFAs have been identified. ${ }^{4-6}$ Diagnosis based on VLCFA measurement only would fail to detect such patients. Analyzing the biosynthesis of ether phospholipids, including plasmalogens, can help to further differentiate between patients from group I or II: a deficient plasmalogen synthesis points to a PBD (group I), whereas normal concentrations of plasmalogens are indicative for a single enzyme/protein deficiency (group II). Nevertheless, only measurement of di- and trihydroxycholestanoic acid, pristanic as well as phytanic acid in plasma can allow to clearly distinguish whether a patient suffers from a PBD or a single enzyme/protein deficiency. Extensive biochemical flowcharts are well established for the distinct diagnosis of every single enzyme/protein deficiency. ${ }^{1}$ But after initial biochemical screening, genetic heterogeneity remains the obstacle for a conclusive diagnosis in PBDs.

In the past two decades, at least 13 different human PEX genes have been identified to be involved in the biogenesis of peroxisomes (group I). These findings led to defining the primary genetic defect in all 13 known complementation groups. ${ }^{7}$ For RCDP, clinical pre-sorting of patients is possible because of characteristic dysmorphic features like shortening of the proximal limbs. The primary genetic defect is restricted to mutations in the PEX7 gene. ${ }^{8}$ In contrast, ZSS patients cannot be assorted clinically. These patients contain mutations in a whole variety of $P E X$ genes, namely PEX1, PEX2, PEX3, PEX5, PEX6, PEX10, PEX12, PEX13, PEX14, PEX16, PEX19 and PEX26. ${ }^{2,9}$ Molecular analysis for ZSS patients ${ }^{3,10}$ is beneficial for genetic counselling, carrier testing or prenatal diagnosis. Furthermore, knowledge of the precise molecular defect in PEX1 contributes to at least some prediction of the clinical course. However, molecular analysis can be problematic because of the limited time and the economic resources available. Even though DNA analysis by direct sequencing is a sophisticated and fast method, sequencing of 12 different and to some extent large genes is still timeconsuming and costly. Efforts on categorizing ZSS patients biochemically have shown that on an average, VLCFA oxidation deficiency is most severe in ZS patients, less pronounced in NALD patients and mildest in IRD patients. ${ }^{1}$ Nevertheless, concentrations of VLCFA are too variable to allow the prediction of clinical course or to point to a certain disease gene. In 2004, a seven-step algorithm based on direct sequencing of distinct exons from six individual PEX genes (PEX1, PEX2, PEX6, PEX10, PEX12 and PEX26) was presented for the molecular diagnosis of ZSS patients. ${ }^{11}$ In a maximum of 14 reactions per patient, this approach was able to identify one pathological mutation in $79 \%$ and both mutant alleles in $58 \%$ of screened patients. To detect the molecular defect in every PBD patient, we developed a new diagnostic strategy for ZSS patients by combining cell biology and molecular genetic methods including all the 12 PEX genes in question. By applying this rational and systematic new approach, we were able to identify the primary genetic defects in our cohort of ZSS patients and manage cost and time efficiently.

\section{Materials and methods}

Methods for measurement of VLCFA have been published by Moser and co-workers in 1980 and $1981 .{ }^{12,13}$

\section{Culture of patient fibroblast cells}

The patient fibroblast cell lines were cultured in Dulbecco's modified Eagle's medium (low glucose) supplemented with $10 \%$ fetal bovine serum and 1\% L-glutamate under $5 \% \mathrm{CO}_{2}$ at $37^{\circ} \mathrm{C}$. Chorionic villous fibroblasts and amniocytes were grown in AmnioGrow Plus (Cytogen, Sinn-Fleisbach, Germany).

\section{Immunofluorescence analysis}

Cells grown on cover slips were fixed with $4 \%$ paraformaldehyde in PBS for $30 \mathrm{~min}$. Then, the cells were washed thrice with PBS and permeabilized with 1\% Triton X-100 in PBS for $5 \mathrm{~min}$. After washing, cells were incubated with rabbit anti-catalase antibody (1:1200 dilution; Oxis, International Inc., Foster City, CA, USA) for $30 \mathrm{~min}$. Subsequently, the cells were washed again and incubated with anti-rabbit antibody labelled with fluorescent dye Cy3 (1:1000 dilution; Dianova GmbH, Hamburg, Germany) for $30 \mathrm{~min}$. Finally, the cells were mounted with ProLong Gold antifade reagent (Invitrogen $\mathrm{GmbH}$, Karlsruhe, Germany) and analyzed under an Axio Imager M1 fluorescence microscope (Carl Zeiss MicroImaging GmbH, Göttingen, Germany).

\section{Complementation by transfection}

Cultured primary human fibroblasts were transfected with pcDNA3 plasmids containing the respective $P E X$ genes (PEX1, PEX2, PEX3, PEX5, PEX6, PEX10, PEX12, PEX13, PEX14, PEX16, PEX19 and PEX26). The commercially available diverse transfection reagents were compared for high transfection efficiency as well as for cell viability of human primary fibroblasts. Microscopic analysis of 
transfected cells indicated the cationic liposome reagent Effectene (Qiagen, Hilden, Germany) as the most suitable. Before the transfection, $4 \times 10^{4}$ fibroblast cells were seeded in 12-well plates. The next day, $0.15 \mu \mathrm{g} P E X$ expression constructs and $0.15 \mu \mathrm{g}$ eGFP-SKL-containing PTS1 (peroxisomal targeting signal 1) were diluted with buffer EC to a total volume of $75 \mu \mathrm{l}$. Cotransfection with eGFP-SKL enables direct visualization of PTS1-dependent import of eGFP in the patient fibroblasts. The Enhancer $(2.4 \mu \mathrm{l})$ and Effectene $(2.4 \mu \mathrm{l})$ were subsequently added to the DNA and the transfection was performed following essentially the instructions of the manufacturer. After $6 \mathrm{~h}$, cells were washed once with PBS and fresh growth medium was added. Transfected cells were further incubated for $48 \mathrm{~h}$ and the localization of catalase was determined through immunofluorescence analysis.

\section{Mutation detection}

Genomic DNA was purified from fibroblasts using DNeasy Tissue Kit or from EDTA whole blood samples using QIAamp DNA Blood Mini Kit (Qiagen). Similarly, total RNA was extracted from patient fibroblast cells with RNeasy Mini Kit (Qiagen). Then, cDNA was synthesized from $2 \mu \mathrm{g}$ RNA using SuperScript III First-Strand Synthesis System for RT-PCR (Invitrogen). Complete ORF of respective $P E X$ transcripts was amplified and sequenced on an ABI PRISM 3100 Avant (Applied Biosystems, Foster City, CA, USA). All identified mutations were confirmed by direct sequencing of two individual PCR amplification products on forward and reverse strands. Primer sequences as well as PCR and sequencing conditions are available on request.

\section{Results and discussion}

We have established a new cost- and time-efficient diagnostic strategy for ZSS patients by combining cell biology and molecular genetic methods including all the 12 PEX genes in question (Figure 1). By applying this systematic approach, we determined the primary genetic defect in all 87 ZSS patients studied. We identified unambiguous mutations in six different PEX genes (Table 1). Some of the novel mutations were published recently. ${ }^{3,14}$ In addition, we identified two new deletion mutations and three new missense mutations in PEX6 (Table 1).

\section{Biochemical analysis: measurement of VLCFA and plasmalogen}

Traditionally, levels of VLCFA and various polyunsaturated fatty acids in serum or plasma were measured to determine whether the patient suffers from peroxisomal defect or not. In ZSS patients, higher levels of VLCFA, notably C26:0, are frequently detected, as peroxisomal function is defective and $\beta$-oxidation of these fatty acids cannot take place properly. In contrast, plasmalogen levels are often reduced in ZSS patients, as biosynthesis of these ether phospholipids is impaired. EDTA whole blood sample necessary for such biochemical analysis can be easily obtained, and a large number of samples can be handled at the same time. Thus, the measurement of VLCFA and plasmalogen provides a suitable parameter for routine screening that points the diagnosis in the right direction. However, it should be kept in mind that cases have been reported where normal VLCFA levels were measured in patients suffering clearly from a peroxisomal disorder ${ }^{4-6}$ and thus, a reliable diagnosis of ZSS cannot be deduced from the quantification of VLCFA only. When patient samples contain a normal level of VLCFA and/or plasmalogen but strong clinical symptoms indicative of ZSS persist, diagnosis of ZSS should not be dismissed altogether but rather, skin biopsy should be undertaken and the follow-up analysis should be performed accordingly (Figure 1).

\section{Sequence analysis of PEX1}

In our screening, the mutation in PEX1 was responsible for peroxisomal defects in nearly $80 \%$ (70 out of 87 ) of patients (Table 1). Novel PEX1 mutations that we identified through this screening were presented earlier. ${ }^{3}$ Several complementation studies and sequencing of patient DNAs have shown that over $60 \%$ of ZSS patients contain mutations in PEX1 gene. ${ }^{3,11,15-17}$ The two most frequently detected mutations are: (i) c.2528G $>$ A missense mutation in exon 15 leading to G843D PEX1 protein and (ii) c.2087_2098ins T insertion mutation in exon 13 leading to truncated I700Yfs PEX1. Therefore, the patients with elevated VLCFA and reduced plasmalogen were first sequenced for these two common mutations in the PEX1 gene. When the patient contained one heterozygous allele of either mutation, PEX1 was completely sequenced to identify the second mutation. In our cohort, 17 ZSS patients would have remained without a definite diagnosis.

\section{Immunofluorescence analysis with anti-catalase antibody}

As all ZSS patients suffer from defects in peroxisomal function, localization of peroxisomal matrix proteins such as catalase in the patient's fibroblast cells can elucidate whether the patient is affected by a PBD. In primary human fibroblasts of patients unaffected by a PBD, catalase is imported properly into the peroxisomal matrix and gives a characteristic punctate pattern in immunofluorescence studies (Figure 2a). In contrast, catalase is diffused in the cytosol of ZSS patient fibroblasts (Figure 2b). In rare cases, patient fibroblasts display a mosaic pattern of catalase localization, that is, partly in the peroxisome as well as in the cytosol (Figure 2c). ${ }^{5,18,19}$ Therefore, immunofluorescence analysis with an antibody against catalase was performed in the fibroblasts of patients whose clinical symptoms indicated ZSS but did not contain mutations in 


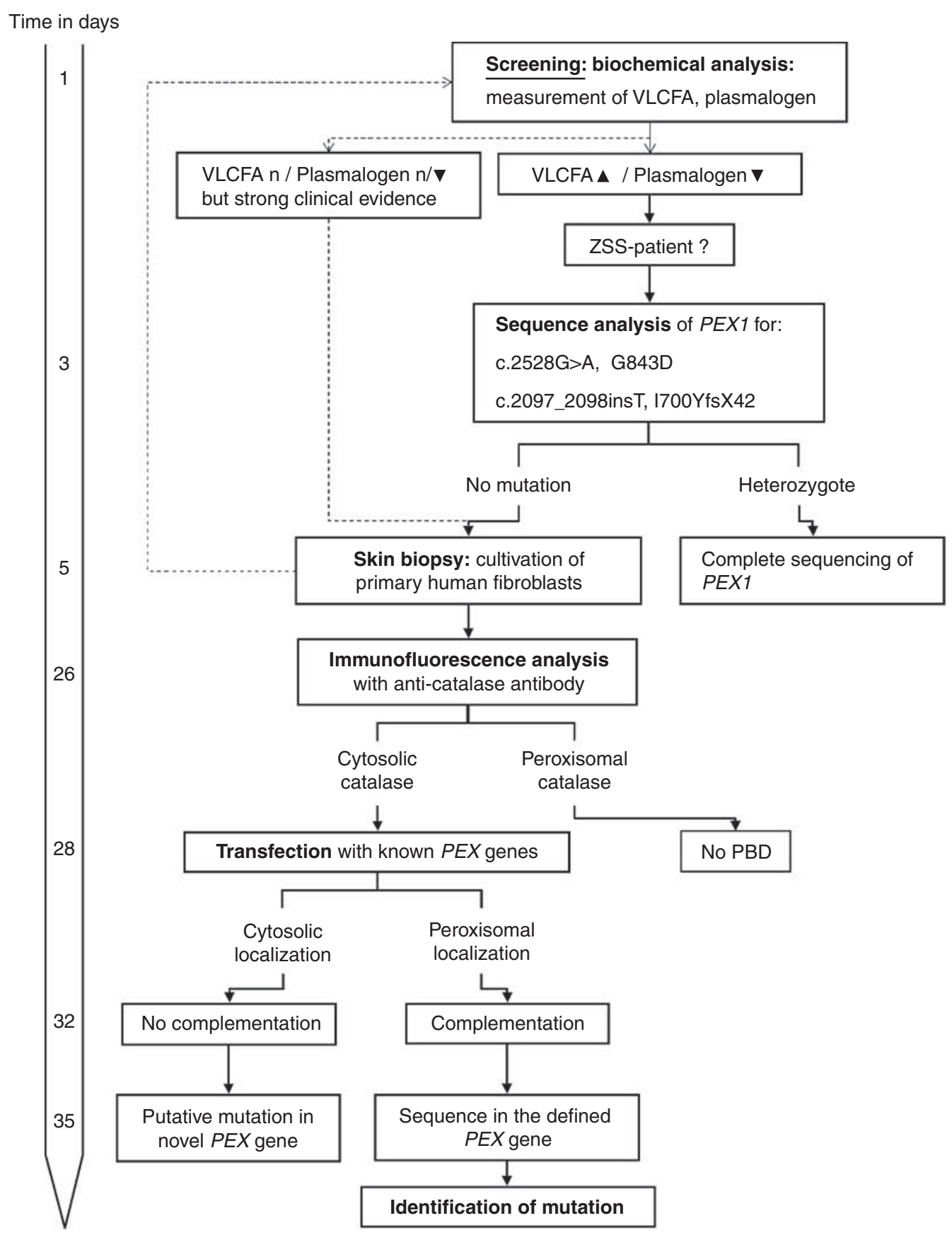

Figure 1 Cost- and time-efficient diagnostic strategy for ZSS. Patients suspected of ZSS are screened by measurement of the concentration of very long chain fatty acids (VLCFAs) in serum/plasma and biosynthesis of plasmalogens in erythrocytes. If VLCFAs are elevated $(\boldsymbol{\Lambda})$ and plasmalogens are decreased $(\boldsymbol{\nabla})$, the patient's DNA from EDTA whole blood sample will be extracted and sequenced for the two most common mutations in the most frequently affected PEX gene, PEX1. If the patient is heterozygotic for one of these two mutations, the entire PEX1 gene will be sequenced. If none of these two mutations are detected, we recommend cultivation of primary human fibroblasts (PHFs). To confirm the suspected diagnosis of ZSS on the cellular level, PHFs are analyzed by immunofluorescence with an anti-catalase antibody. If catalase is localized inside the peroxisome (see Figure 2a), ZSS is ruled out in the patient. If catalase is diffused in the cytosol of the patient's PHFs, diagnosis of ZSS is verified. Then, the patient's PHFs are transfected with all known human PEX genes (except PEX7) cloned in individual expression vectors and together with eGFP-SKL to screen for complementation. The PEX gene that complements the patient's PHFs will be sequenced. If none of the known human PEX genes complements the patient's PHFs, the patient potentially belongs to an unknown complementation group with a putative mutation in a novel, and so far unidentified PEX gene. The time bar on the left displays the approximate estimation of time needed for the different laboratory procedures used in this strategy. If concentrations of VLCFAs are within the normal range $(n)$ and biosynthesis of plasmalogens in erythrocytes is also within the normal range or is only slightly decreased, but clinical presentation strongly points to a PBD, we recommend a skin biopsy to culture PHFs $(\rightarrow)$. Measurement of the concentration of VLCFAs, as well as biosynthesis of plasmalogens, should be repeated in these PHFs. 
Table 1 The unambiguous mutations identified in six different PEX genes

\begin{tabular}{|c|c|c|c|c|c|c|}
\hline PEX gene & Mutation: cDNA level & Exon & $\begin{array}{l}\text { Mutation: protein } \\
\text { level }\end{array}$ & $\begin{array}{l}\text { Number of } \\
\text { alleles }\end{array}$ & References & $\begin{array}{l}\text { Detected }(d) / \text { not detected }(n d) \\
\text { Steinberg et al } \text { l }^{11}\end{array}$ \\
\hline PEX1 & $\begin{array}{l}\text { c. } 274 G>C \\
\text { c. } 911 \_912 \text { delCT } \\
\text { c. } 1865 \_1866 \text { in- } \\
\text { sGAGTGTGGA } \\
\text { c. } 2083 \_2085 \text { delATG } \\
\text { c. } 2097 \_2098 \text { insT } \\
\text { c. } 2227 \_2416 \text { del190bp } \\
\text { c. } 2387 T>C \\
\text { c. } 2528 \mathrm{C}>\mathrm{A} \\
\text { c. } 2614 \mathrm{C}>\mathrm{T} \\
\text { c. } 2814 \_2818 \text { delCTTTG } \\
\text { c. } 3038 \mathrm{G}>\mathrm{A} \\
\text { c. } 3287 \mathrm{C}>\mathrm{G} \\
\text { c. } 3378 \mathrm{C}>\mathrm{G} \\
\text { c. } 3691 \_3694 \text { delCAGT }\end{array}$ & $\begin{array}{r}4 \\
5 \\
11 \\
13 \\
13 \\
14 \\
14 \\
15 \\
16 \\
18 \\
20 \\
21 \\
21 \\
23\end{array}$ & $\begin{array}{l}\text { p.V92L } \\
\text { p.S304CfsX4 } \\
\text { p.A622_H623insSVC } \\
\text { p.M695del } \\
\text { p.I700YfsX42 } \\
\text { p.E743NfsX2 } \\
\text { p.L796P } \\
\text { p.G843D } \\
\text { p.R872X } \\
\text { p.F938LfSX2 } \\
\text { p.R1013H } \\
\text { p.S1096X } \\
\text { p.Y1126X } \\
\text { p.Q1231HfsX3 }\end{array}$ & $\begin{array}{r}2 \\
1 \\
2 \\
\\
2 \\
52 \\
3 \\
1 \\
68 \\
3 \\
1 \\
1 \\
1 \\
1 \\
2\end{array}$ & 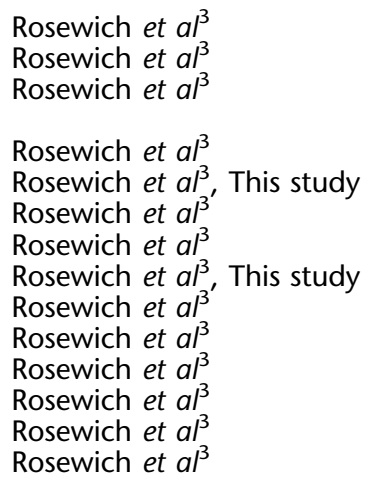 & $\begin{array}{l}\text { nd } \\
\text { nd } \\
\text { nd } \\
\text { d } \\
d \\
\text { nd } \\
\text { nd } \\
\text { d } \\
\text { nd } \\
\text { d } \\
\text { nd } \\
\text { nd } \\
\text { nd } \\
\text { nd }\end{array}$ \\
\hline PEX2 & $\begin{array}{l}\text { c. } 115 \mathrm{C}>\mathrm{T} \\
\text { c.834_838delTACTT }\end{array}$ & $\begin{array}{l}3 \\
3\end{array}$ & $\begin{array}{l}\text { p.Q39X } \\
\text { p.F278LfsX3 }\end{array}$ & $\begin{array}{l}2 \\
4\end{array}$ & $\begin{array}{l}\text { Krause et } a l^{14} \\
\text { Krause et } a l^{14}\end{array}$ & $\begin{array}{l}d \\
d\end{array}$ \\
\hline PEX6 & $\begin{array}{l}\text { c. } 685 \text { 686insAG } \\
\text { c. } 1314 \_1321 \\
\text { delGGAGGCCT } \\
\text { c. } 1415 \text { dupC } \\
\text { c. } 1946 \text { delG } \\
\text { c. } 2048 T>C \\
\text { c. } 2120 T>\text { G } \\
\text { c. } 2362 \mathrm{G}>\mathrm{A} \\
\text { c. } 2426 \mathrm{C}>\mathrm{T} \\
\text { c. } 2434 \mathrm{C}>\mathrm{T}\end{array}$ & $\begin{array}{r}1 \\
5 \\
\\
6 \\
9 \\
10 \\
10 \\
11 \\
13 \\
13\end{array}$ & $\begin{array}{l}\text { p.S232HfsX15 } \\
\text { p.E439GfsX3 } \\
\text { p.G473RfsX13 } \\
\text { p.I650SfsX10 } \\
\text { p.L683P } \\
\text { p.V707G } \\
\text { p.V788M } \\
\text { p.A809V } \\
\text { p.R812W }\end{array}$ & $\begin{array}{l}6 \\
2 \\
2 \\
2 \\
1 \\
2 \\
1 \\
1 \\
1\end{array}$ & $\begin{array}{l}\text { Krause et } a l^{14} \\
\text { This study, unpublished } \\
\text { Krause et } a l^{14} \\
\text { This study, unpublished } \\
\text { This study, unpublished } \\
\text { This study, unpublished } \\
\text { This study, unpublished } \\
\text { This study } \\
\text { This study }\end{array}$ & $\begin{array}{l}\mathrm{d} \\
\text { nd } \\
\text { nd } \\
\text { nd } \\
\text { nd } \\
\text { nd } \\
\text { nd } \\
\text { d } \\
\text { d }\end{array}$ \\
\hline PEX10 & $\begin{array}{l}\text { c. } 730 \mathrm{C}>\mathrm{T} \\
\text { c. } 874 \_875 \mathrm{delCT} \\
\text { c. } 881 \mathrm{G}>\mathrm{A}\end{array}$ & $\begin{array}{l}4 \\
4 \\
5\end{array}$ & $\begin{array}{l}\text { p.Arg244X } \\
\text { p.L272fs } \\
\text { p.Trp294X }\end{array}$ & $\begin{array}{l}3 \\
2 \\
1\end{array}$ & $\begin{array}{l}\text { Krause et } a l^{14} \\
\text { This study } \\
\text { Krause et } a l^{14}\end{array}$ & $\begin{array}{l}d \\
d \\
d\end{array}$ \\
\hline PEX12 & c.875_876delCT & 3 & p.Ser292X & 2 & Krause et $a l^{14}$ & $d$ \\
\hline \multirow[t]{2}{*}{ PEX13 } & c.937T > G & 4 & p.Trp313Gly & 2 & Krause et $a l^{14}$ & nd \\
\hline & & & Total & 174 & & \\
\hline
\end{tabular}

PEX1. Patients whose fibroblasts showed exclusively peroxisomal catalase pattern most likely belong to other disease groups and were dismissed from further analysis. Catalase was localized only in the cytosol in 15 patient fibroblasts, whereas mosaic pattern was observed in two patient cell lines. Three cell lines showing peroxisomal catalase only were not investigated further.

\section{Complementation analysis through transfection}

Although subcellular localization of catalase in primary human skin fibroblasts can help verify whether the patient suffers indeed from ZSS or not, it does not provide information regarding which $P E X$ gene is defective as a mutation in any of the 12 PEX genes can impair peroxisomal function. In the past, ZSS patient fibroblasts were often fused with other fibroblasts of known disrupted $P E X$ genes and analyzed for the restoration of catalase in the peroxisome of the heterokaryons. ${ }^{16,17}$ If the defective genes in the two fibroblast cell lines are different, they would be able to complement each other and peroxisomal function would be restored. Consequently, catalase would be found in the peroxisome in the heterokaryons. However, if the disrupted genes are identical, there would be no complementation and only cytosolic catalase would be observed. Such complementation analysis requires various reference patient fibroblast cell lines containing a mutation in each of the PEX genes and is a rather cumbersome process.

An alternative approach is to transfect the patient fibroblasts with expression plasmids containing each of the known human PEX genes. ${ }^{14}$ Cotransfection with eGFP-SKL, a green fluorescent protein (GFP) carrying PTS1 sequence SKL, allows easy estimation of transfection efficiency as well as simple analysis of complementation in living cells. However, we observed in rare cases that eGFPSKL was localized in the peroxisome, whereas catalase was cytosolic (data not shown). This is most likely due to the fact that the peroxisomal targeting signal (KANL) of catalase is less efficient than the SKL 

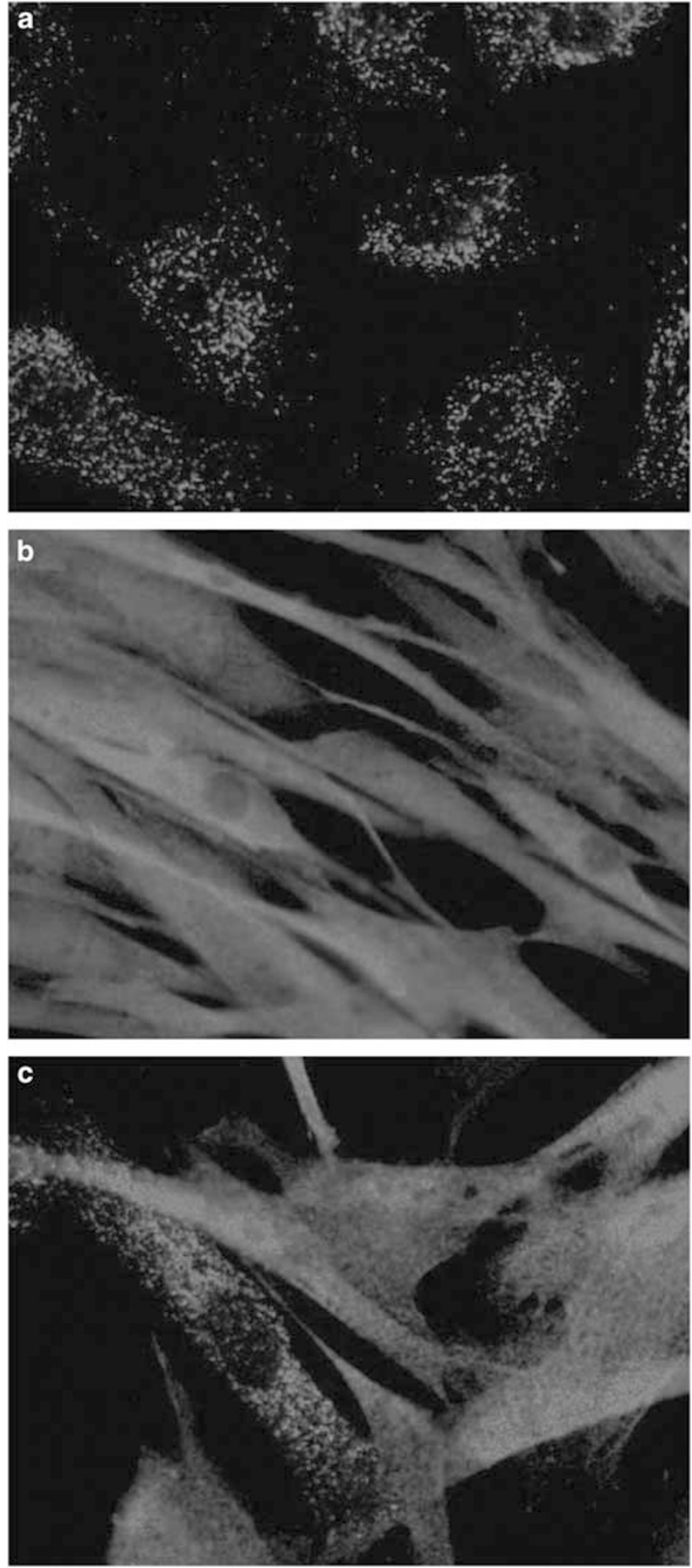

Figure 2 Immunofluorescence analysis of primary human fibroblasts with anti-catalase antibody. (a) In normal human fibroblasts, the peroxisomal matrix protein catalase is localized in the peroxisome as indicated by the punctate pattern. (b) ZSS patient fibroblasts fail to import catalase correctly into the peroxisome and thus show diffused localization of catalase in the cytosol. (c) In rare cases, ZSS patient fibroblasts show mosaicism, in which catalase is localized in the peroxisome as well as in the cytosol. sequence. $^{20,21}$ Consequently, import of catalase seems to be more sensitive to the peroxisomal defect. Overall, we identified unambiguous mutations in six different PEX genes in 87 ZSS patients (Table 1) using this approach. Some of the novel mutations were published recently. ${ }^{3,14}$ In addition, we identified two new deletion mutations and three new missense mutations in PEX6 (Table 1).

\section{Advantages of identifying the molecular defect in a ZSS patient}

In most cases, the diagnosis of ZSS can be established by characteristic clinical, biochemical and cellular patterns, and identification of pathological mutation is not mandatory for the diagnosis. Nevertheless, knowing the underlying primary genetic defect is beneficial for carrier testing and prenatal diagnosis, as the DNA analysis of a fetus at risk provides the most straightforward prenatal diagnosis. So far, it could not be performed for every case, as the precise molecular defect had not been identified for every ZSS patient due to the limitation in resources and/or time. Therefore, prenatal diagnosis of ZSS relies often on the measurement of VLCFA content and/or plasmalogen synthesis in cultured chorionic villus cells or amniocytes. ${ }^{11,22,23}$ However, biochemical analysis in cultured cells was shown to be especially susceptible to the contamination by maternal cells or microorganisms endangering the accuracy of the diagnosis ${ }^{11}$, and alternative methods, such as immunofluorescence analysis using antibodies against catalase, have been applied in parallel. ${ }^{14}$

Although there are 31 PEX genes identified in yeast, only 13 PEX genes have been found in humans so far. As most of the $P E X$ genes were shown to be conserved from yeast to humans, there are probably additional human PEX genes that are yet unknown. In the past years, human PEX genes have been identified either through a homology search of the human EST database using yeast $P E X$ gene sequences or by searching for cDNAs that can functionally complement CHO mutants defective in peroxisomal biogenesis. ${ }^{9}$ Determination of $P E X$ gene mutations by applying the diagnostic strategy presented in this study may help identify ZSS patients who cannot be complemented by known PEX proteins as they contain a pathological mutation in novel PEX genes.

\section{Efficiency in time and cost for determining mutations in ZSS patients}

Apparently, it is not a simple task to identify the pathogenic mutation when the most common mutations in PEX1 have been ruled out. Thus, mutations have remained unknown in many patients who were clinically and biochemically diagnosed with ZSS. Applying the recently introduced PEX Gene Screen Algorithm, at least some exons of six PEX genes (PEX1, PEX2, PEX6, PEX10, $P E X 12$ and $P E X 26$ ) can be screened for putative pathogenic mutation. Applying this PEX Gene Screen Algorithm to our 
cohort would have instead detected only 145 out of 172 alleles in those six PEX genes, leaving 15 out of 87 patients (17\%) without a definite diagnosis (the last column in Table 1). As the PEX Gene Screen Algorithm does not include PEX13 in its hierarchical sequencing, it would have missed the pathogenic mutation that enabled the subsequent prenatal diagnosis. Furthermore, diverse reports of mutations in PEX genes show that such a strategy fails to identify critical molecular defects underlying the clinical manifestation in many ZSS patients. ${ }^{3,5,10,24,25}$

As the exact knowledge about the pathogenic mutation in the patient provides valuable information, we were interested in strategies that could detect PEX gene defects in every single ZSS patient within a reasonable time and cost. On applying the published PEX Gene Screen Algorithm, the mutation could not be identified in $21 \%$ of the screened ZSS patients ${ }^{11}$. To search for the molecular defects further in these remaining $21 \%$ of patients, all other PEX exons should be sequenced one after another. Estimating the cost of PCR and sequencing per reaction as $100-300 €$, which will vary strongly depending on the laboratory's facility and its location, and assuming at least 70 PCR reactions to cover all 12 PEX genes, identifying the causative mutation in a ZSS patient can cost 7000-21000€. As $\mathrm{PCR} /$ sequencing reaction is performed in a hierarchical system, in which the subsequent step depends on the result of the earlier sequencing reaction, the whole procedure may take several weeks until the mutation is found finally.

On the other hand, when fibroblast cultures from patients are available, the fibroblast cells can be complemented to efficiently identify the defective PEX gene first. Once it is clear which PEX gene most likely contains the pathogenic mutation, it requires only two to six PCRs or sequencing reactions to locate the molecular defect in that specific PEX gene. Estimating the cost of complementation and the subsequent immunofluorescence analysis with an anticatalase antibody as approximately $700 €$ per patient, a maximum of $2500 €$ may be sufficient to identify the mutation in a ZSS patient. Although it may seem cumbersome to establish the fibroblast culture, it should be noted that more and more studies recommend biochemical and/or immunofluorescence analysis in fibroblast cells for accurate diagnosis of ZSS. Once the fibroblast culture is set up, which may take 10 days to 3 weeks, the complementation and the subsequent sequencing analysis can be performed efficiently within a week. Thus, when patient fibroblast cells are available, the diagnostic strategy applying a systematic combination of biochemical analysis, sequencing and complementation through transfection may provide a more efficient approach than the hierarchical sequencing. In summary, we developed an alternative strategy that detected successfully the underlying genetic defect in all ZSS patients screened in this study. This new strategy will provide valuable benefits for other already known and future ZSS patients with yet unresolved molecular causes.

\section{Acknowledgements}

This work was supported by the Grant GA 354/5-1 und 5-2 from the Deutsche Forschungsgemeinschaft, DFG (JG) and by the Research program of the Faculty of Medicine, Georg-August-University Göttingen (HR).

\section{References}

1 Wanders RJ: Metabolic and molecular basis of peroxisomal disorders: a review. Am J Med Genet A 2004; 126: 355-375.

2 Matsumoto N, Tamura S, Furuki S et al: Mutations in nove peroxin gene PEX26 that cause peroxisome-biogenesis disorders of complementation group 8 provide a genotype-phenotype correlation. Am J Hum Genet 2003; 73: 233-246.

3 Rosewich H, Ohlenbusch A, Gartner J: Genetic and clinical aspects of Zellweger spectrum patients with PEX1 mutations. J Med Genet 2005; 42: e58.

4 Wanders RJ, Waterham HR: Peroxisomal disorders I: biochemistry and genetics of peroxisome biogenesis disorders. Clin Genet 2005; 67: $107-133$.

5 Zeharia A, Ebberink MS, Wanders RJ et al: A novel PEX12 mutation identified as the cause of a peroxisomal biogenesis disorder with mild clinical phenotype, mild biochemical abnormalities in fibroblasts and a mosaic catalase immunofluorescence pattern, even at 40 degrees C. J Hum Genet 2007; 52: 599-606.

6 Rosewich H, Waterham HR, Wanders RJ et al: Pitfall in metabolic screening in a patient with fatal peroxisomal beta-oxidation defect. Neuropediatrics 2006; 37: 95-98.

7 Steinberg SJ, Dodt G, Raymond GV, Braverman NE, Moser AB, Moser HW: Peroxisome biogenesis disorders. Biochim Biophys Acta 2006; 1763: 1733-1748.

8 Purdue PE, Zhang JW, Skoneczny M, Lazarow PB: Rhizomelic chondrodysplasia punctata is caused by deficiency of human PEX7, a homologue of the yeast PTS2 receptor. Nat Genet 1997; 15: $381-384$.

9 Weller S, Gould SJ, Valle D: Peroxisome biogenesis disorders. Annu Rev Genomics Hum Genet 2003; 4: 165-211.

10 Maxwell MA, Allen T, Solly PB, Svingen T, Paton BC, Crane DI: Novel PEX1 mutations and genotype-phenotype correlations in Australasian peroxisome biogenesis disorder patients. Hum Mutat 2002; 20: 342-351.

11 Steinberg S, Chen L, Wei L et al: The PEX Gene Screen: molecular diagnosis of peroxisome biogenesis disorders in the Zellweger syndrome spectrum. Mol Genet Metab 2004; 83: 252-263.

12 Moser HW, Moser AB, Kawamura N et al: Adrenoleukodystrophy: elevated C26 fatty acid in cultured skin fibroblasts. Ann Neurol 1980; 7: 542-549.

13 Moser HW, Moser AB, Frayer KK et al: Adrenoleukodystrophy: increased plasma content of saturated very long chain fatty acids. Neurology 1981; 31: 1241-1249.

14 Krause C, Rosewich H, Thanos M, Gartner J: Identification of novel mutations in PEX2, PEX6, PEX10, PEX12, and PEX13 in Zellweger spectrum patients. Hum Mutat 2006; 27: 1157.

15 Reuber BE, Germain-Lee E, Collins CS et al: Mutations in PEX1 are the most common cause of peroxisome biogenesis disorders. Nat Genet 1997; 17: 445-448.

16 Moser AB, Rasmussen M, Naidu S et al: Phenotype of patients with peroxisomal disorders subdivided into sixteen complementation groups. J Pediatr 1995; 127: 13-22.

17 Wanders RJ, Mooijer PA, Dekker C, Suzuki Y, Shimozawa N: Disorders of peroxisome biogenesis: complementation analysis shows genetic heterogeneity with strong overrepresentation of one group (PEX1 deficiency). J Inherit Metab Dis 1999; 22: 314-318.

18 Gootjes J, Schmohl F, Mooijer PA et al: Identification of the molecular defect in patients with peroxisomal mosaicism using a novel method involving culturing of cells at 40 degrees C: implications for other inborn errors of metabolism. Hum Mutat 2004; 24: $130-139$ 
19 Shimozawa N, Imamura A, Zhang Z et al: Defective PEX gene products correlate with the protein import, biochemical abnormalities, and phenotypic heterogeneity in peroxisome biogenesis disorders. J Med Genet 1999; 36: 779-781.

20 Purdue PE, Castro SM, Protopopov V, Lazarow PB: Targeting of human catalase to peroxisomes is dependent upon a novel C-terminal peroxisomal targeting sequence. Ann N Y Acad Sci 1996; 804: 775-776.

21 Legakis JE, Koepke JI, Jedeszko C et al: Peroxisome senescence in human fibroblasts. Mol Biol Cell 2002; 13: 4243-4255.

22 Roscher A, Molzer B, Bernheimer H, Stockler S, Mutz I, Paltauf F: The cerebrohepatorenal (Zellweger) syndrome: an improved method for the biochemical diagnosis and its potential value for prenatal detection. Pediatr Res 1985; 19: 930-933.

23 Wanders RJ, Schutgens RB, van den Bosch H, Tager JM, Kleijer WJ Prenatal diagnosis of inborn errors in peroxisomal beta-oxidation. Prenat Diagn 1991; 11: 253-261.

24 Walter C, Gootjes J, Mooijer PA et al: Disorders of peroxisome biogenesis due to mutations in PEX1: phenotypes and PEX1 protein levels. Am J Hum Genet 2001; 69: 35-48.

25 Zhang Z, Suzuki Y, Shimozawa N et al: Genomic structure and identification of 11 novel mutations of the PEX6 (peroxisome assembly factor-2) gene in patients with peroxisome biogenesis disorders. Hum Mutat 1999; 13: 487-496. 